\title{
A nutrient profiling assessment of packaged foods using two star-based front-of-pack labels
}

\author{
Amy M Carrad', Jimmy Chun Yu Louie ${ }^{2, *}$, Heather R Yeatman ${ }^{1}$, Elizabeth K Dunford ${ }^{3}$, \\ Bruce $\mathrm{C} \mathrm{Neal}^{3}$ and Victoria M Flood ${ }^{4}$ \\ ${ }^{1}$ School of Health \& Society, University of Wollongong, Wollongong, New South Wales, Australia: ${ }^{2}$ School of \\ Molecular Bioscience, Faculty of Science, University of Sydney, Level 4 East, The Hub, D17 Charles Perkins Centre, \\ The University of Sydney, NSW 2006, Australia: ${ }^{3}$ The George Institute for Global Health, Royal Prince Alfred \\ Hospital, Camperdown, New South Wales, Australia: ${ }^{4}$ Faculty of Health Sciences, University of Sydney, Lidcombe, \\ New South Wales, Australia
}

Submitted 2 February 2015: Final revision received 19 August 2015: Accepted 24 August 2015: First published online 28 September 2015

\begin{abstract}
Objective: To compare two front-of-pack nutrition labelling systems for the assessment of packaged foods and drinks with Australian Dietary Guidelines.

Design: A cross-sectional nutrient profiling assessment. Food and drink products ( $n$ 20 225) were categorised into scoring levels using criteria for the Institute of Medicine (IOM) three-star system and the five-star Australian Health Star Rating (HSR). The effectiveness of these systems to categorise foods in accordance with Australian Dietary Guidelines was explored.

Setting: The study was conducted in Australia, using a comprehensive food database. Subjects: Packaged food and drink products ( $n$ 20 225) available in Australia. Results: Using the IOM three-star system, the majority (55\%) of products scored the minimum 0 points and $25.5 \%$ scored the maximum 3 points. Using HSR criteria, the greatest proportion of products (15.2\%) scored three-and-a-half stars from a possible five and $12.5 \%$ received the lowest rating of a half-star. Very few products $(4 \cdot 1 \%)$ scored five stars. Products considered core foods and drinks in Australian Dietary Guidelines received higher scores than discretionary foods in all food categories for both labelling systems (all $P<0 \cdot 05$; Mann-Whitney $U$ test), with the exception of fish products using IOM three-star criteria $(P=0 \cdot 603)$. The largest discrepancies in median score between the two systems were for the food categories edible oils, convenience foods and dairy.

Conclusions: Both the IOM three-star and Australian HSR front-of-pack labelling systems rated packaged foods and drinks broadly in line with Australian Dietary Guidelines by assigning core foods higher ratings and discretionary foods lower ratings.
\end{abstract}

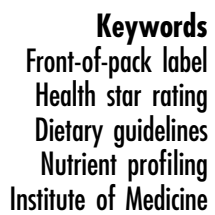

Internationally, the prevalence of overweight and obesity among children and adults has increased in recent decades $^{(1)}$. Excessive intake of energy-dense foods is one factor that contributes to the development of obesity and a range of non-communicable diseases ${ }^{(2)}$. To reduce the risk of these diseases, as in many other countries, the Australian Dietary Guidelines (ADG) recommend a diet containing a variety of foods from the core groups - grain or cereal foods, fruits, vegetables, reduced-fat dairy products, and lean meats and alternatives - and limiting the intake of discretionary products containing high amounts of saturated fat, added sugars and added salt ${ }^{(3)}$. Paradoxically, many of the foods readily available in supermarkets are ultra-processed, energy-dense products that contain high amounts of these nutrients that are linked to adverse health conditions ${ }^{(2,3)}$.

In Australia, it is mandatory for these packaged foods and drinks to display a nutrition information panel, designed to provide consumers with a technical description of the nutritional content ${ }^{(4)}$. However, issues of consumer health literacy and numeracy mean that such labelling may not effectively allow individuals to make informed choices about which foods to purchase and consume $^{(5)}$. Consequently, various methods of nutrient profiling have been developed for usage in monitoring health claims made on foods, regulating food advertising and designing front-of-pack labelling (FOPL) ${ }^{(6)}$. Examples include Guideline Daily Amounts (multinational), Traffic 
Light labelling and the WXYfm/Ofcom model (UK), the Keyhole logo (Sweden), the Nutrient Profiling Scoring Criterion (Australia and New Zealand), the Choices logo (Netherlands) and NuVal (USA) ${ }^{(6,7)}$.

FOPL is intended to provide consumers with an easily comprehensible summary of the nutritional quality of foods and drinks, allowing rapid comparisons between products at the point of purchase ${ }^{(8)}$. At present, FOPL is voluntary in Australia, with a number of systems in use, including the Daily Intake Guide and the Heart Foundation $\operatorname{Tick}^{(9,10)}$. In 2011, recommendations were made for the introduction of an interpretive FOPL system, such as the Multiple Traffic Light scheme ${ }^{(11)}$. Previous research has shown traffic light FOPL to be effective at enabling consumers to select the healthier option when presented with a set of foods ${ }^{(8,12)}$.

Following further negotiations with stakeholders, policy for an alternative FOPL guide in Australia was announced in June $2013^{(13)}$. The proposed FOPL system was the Health Star Rating (HSR), which was to be initially implemented as a voluntary system ${ }^{(13)}$. The HSR algorithm was based on a modified Nutrient Profiling Scoring Criterion (NPSC), which is used to determine whether a food product is eligible to carry a health claim, such as statements linking the benefits of calcium to bone health ${ }^{(14)}$. Under the HSR, packaged foods and drinks may receive from a half-star to five stars based on energy, saturated fat, sodium, sugars, and fibre or protein (Fig. 1). The HSR is similar in presentation to a three-star FOPL system developed by the Institute of Medicine (IOM) by which products may be awarded $0,1,2$ or 3 points depending on their saturated fat, trans fat, added sugars and sodium content (Fig. 1) ${ }^{(15)}$.

It is important that any FOPL system presents nutrition information in such a way that is aligned with the central

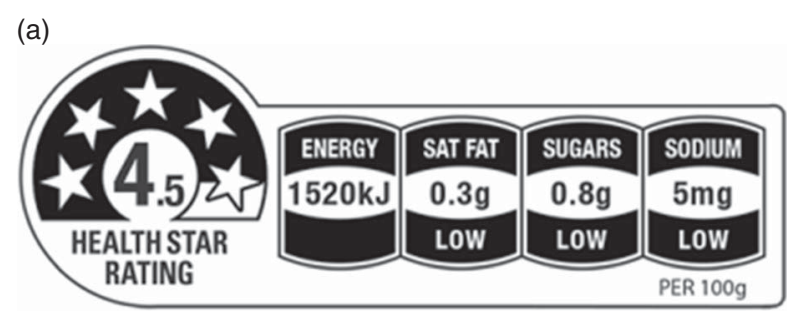

(b)

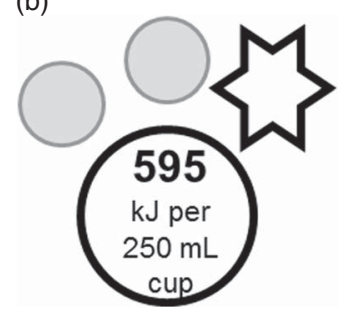

Fig. 1 Example of (a) the Australian Health Star Rating and (b) the Institute of Medicine three-star front-of-pack food labelling systems messages of national dietary guidelines in order to provide consumers with consistent messages. Methods for assessing the validity of nutrient profiling schemes have been outlined in a technical report produced by the $\mathrm{WHO}^{(16)}$. Validity assessment seeks to confirm if a nutrient profiling model correctly classifies foods. One such method is to compare the ratings received by foods using the given model with existing dietary guidelines ${ }^{(16)}$.

No research has been published on how the HSR or IOM three-star FOPL systems would be represented on packaged foods and drinks available through the Australian food supply. The present study aims to examine how packaged food and beverage products in Australia would be rated using these two systems and to test for differences between the FOPL systems at the food category level. It also aims to investigate whether the systems reflect recommendations of the ADG which categorises foods as core (vegetables; fruit; grain/cereal foods; lean meats and substitutes; dairy) or discretionary ${ }^{(3)}$.

\section{Methods}

The George Institute for Global Health's branded food composition database was used for this project. Methods have been described elsewhere ${ }^{(17)}$, but in brief this database contains annually updated nutritional information for more than 20000 processed food products from Australian supermarkets collected from nutrition information panels. This database does not contain information on fresh produce, such as fruits and vegetables, as these products are not obliged to display nutrition information ${ }^{(4)}$. The 2012 version of the database used for the present study contained 26509 items in sixty-two broad food categories, and sixteen more specific food categories were used (see online supplementary material, Supplemental Table 1 for a listing of food categories). Products in the database were assessed against nutrient profiling criteria for two FOPL systems, the Australian HSR system and the IOM three-star system. These two systems were selected for inclusion in the study as both have similar star-based formats that are relative across all food types rather than within food groups, yet distinctly varied underlying nutrient assessment methods. An investigation of the applicability of a British nutrient profiling system to a French food composition database has previously been conducted and found that the British Ofcom model did classify foods in the French food supply in such a way that was consistent with the French dietary guidelines ${ }^{(18)}$. The present study utilises a similar approach by applying an American profiling model to an Australian food database.

The methods used have some limitations including: dependency on the accuracy of values on the nutrition information panel of food labels; the use of blanket estimates by food category for added sugars; and the lack of availability of fibre, trans fat, and fruit, vegetable, nut and 
legume (FVNL) content. However, despite these limitations it remains possible to assess the two FOPL systems against the ADG.

\section{Rating food and drink products using the Institute of Medicine three-star system}

The IOM three-star system specifies criteria for saturated fat, trans fat, sodium and added sugars on a per serving basis (Table 1). Information on trans fat content was not available for every food item in the database as it is not a mandatory labelling requirement in Australia; thus trans fat was not assessed in the current study. This may lead to an overestimation of the healthiness of some products such as pastries; however, the amount of trans fat in Australian foods is generally low, with $82 \%$ of processed and takeaway foods containing less than $2 \mathrm{~g}$ of trans-fatty acids per $100 \mathrm{~g}^{(19)}$. A review by Food Standards Australia New Zealand found that the average Australian has an intake of trans fats amounting to $0.5-0.6 \%$ of total daily energy intake, which is below the WHO's recommendation of $1 \%{ }^{(20)}$. Therefore the effect may be negligible but we are unable to formally assess this. Added sugars data were also not available (only total sugars is labelled on all Australian products); however, added sugar values for each product were estimated as a proportion of total sugars by food category using a previously trialled method ${ }^{(21)}$.

The two levels of criteria - 'eligibility' and 'qualifying' of the IOM three-star system were used to determine product ratings (Fig. 2). Food and drink items received a rating of zero to three stars, in whole-star increments. Products were initially assessed against eligibility values for saturated fat, sodium and added sugars (Table 1). Products containing amounts of any one of these nutrients in excess of the levels set were deemed ineligible to receive FOPL points. Food items with sufficiently low quantities of all three nutritional components were then assessed using the secondary qualifying criteria. For each nutritional component with which a product complied with qualifying criteria, the product received a FOPL point.

\section{Rating food and drink products using the Australian Health Star Rating system}

The HSR includes criteria for: saturated fat; total sugars; sodium; energy; fibre; protein; calcium criteria for dairy products; and FVNL content. Calculation of HSR points was performed using criteria from the Draft Guide to the HSR Calculator, which uses an algorithm based on a modified NPSC ${ }^{(22)}$.

The HSR system required products to be categorised into FOPL Calibration Categories (non-dairy beverages; core cereals; core dairy beverages; core dairy cheeses; core dairy yoghurt and soft cheese; fats and oils; fruit; vegetables; protein foods; non-core foods). Food subgroups in the data set were allocated to appropriate FOPL Calibration Categories.

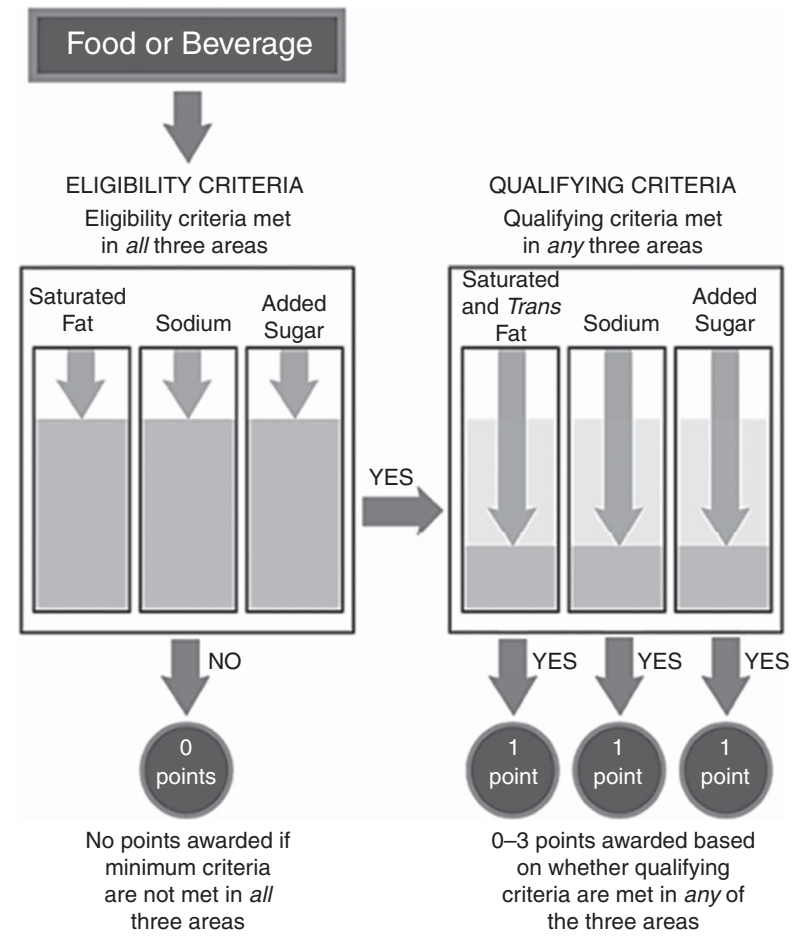

Fig. 2 Process for the evaluation of food and drink products using the Institute of Medicine three-star system ${ }^{(15)}$

Table 1 Nutrient assessment criteria adapted from the Institute of Medicine three-star system

\begin{tabular}{|c|c|c|}
\hline $\begin{array}{l}\text { Nutritional } \\
\text { component }\end{array}$ & Eligibility criteria & Qualifying criteria \\
\hline $\begin{array}{l}\text { Saturated fat } \\
\text { Trans fat }\end{array}$ & $\begin{array}{l}\leq 4 \mathrm{~g} \text { per serving; or per } 50 \mathrm{~g} \text { if serving size } \\
\leq 30 \mathrm{~g} \text { or } \leq 2 \text { tablespoons }\end{array}$ & $\begin{array}{l}\leq 2 \mathrm{~g} \text { per serving } \\
\leq 4.5 \mathrm{~g} \text { per serving and per } 100 \mathrm{~g} \text { for lean seafood and game meats } \ddagger \\
<0.5 \mathrm{~g} \text { per serving; or } \geq 0.5 \mathrm{~g} \text { per serving and product does not contain } \\
\text { partially hydrogenated vegetable oil }\end{array}$ \\
\hline Sodium & $\begin{array}{l}\leq 480 \mathrm{mg} \text { per serving; or per } 50 \mathrm{~g} \text { if serving size } \\
\leq 30 \mathrm{~g} \text { or } \leq 2 \text { tablespoons }\end{array}$ & $\leq 480 \mathrm{mg}$ per serving; or per $50 \mathrm{~g}$ if serving size $\leq 30 \mathrm{~g}$ or $\leq 2$ tablespoons \\
\hline $\begin{array}{l}\text { Added } \\
\text { sugars }\end{array}$ & $\begin{array}{l}\text { Products not categorised as sugars, sweets and } \\
\text { beveragest }\end{array}$ & $\begin{array}{l}\text { Estimated added sugars }=0 \text {; or total sugars content with specified } \\
\text { conditions } \S\end{array}$ \\
\hline
\end{tabular}

†Includes: confectionery; dairy desserts; ice cream and edible ices; jams; beverages; sugars, honey and related products.

fLean seafood contains: $<10 \mathrm{~g}$ total fat per $100 \mathrm{~g}$ and per serving; and $\leq 4.5 \mathrm{~g}$ saturated fat per $100 \mathrm{~g}$ and per serving.

§Qualifying sugars criteria include the following: (i) product contains no estimated added sugars; or (ii) product contains $\leq 5 \mathrm{~g}$ total sugars per serving except for canned products containing tomatoes and/or other vegetables, and yoghurt products; or (iii) canned products that contain $\leq 10 \mathrm{~g}$ total sugars per serving and tomatoes and/or other vegetables containing naturally occurring sugars; or (iv) yoghurt products that contain $\leq 20 \mathrm{~g}$ total sugars per serving. 
In cases where the ingredients of individual products within a sub-category varied greatly (e.g. vegetable-based salads and pasta-salads), FOPL Calibration Categories were determined by product name and ingredients lists. This was the case for the following food categories: baby foods ( $n$ 224); salads and sandwiches ( $n$ 66); flavoured cheeses (e.g. fruit and nut cheeses, $n$ 34); and fruit bars and other fruit ( $n$ 137).

Dairy foods were categorised into FOPL Calibration Categories by calcium content. Dairy-based beverages with greater than $40 \mathrm{mg}$ Ca per $100 \mathrm{ml}$ were classified as core dairy, and were otherwise classified as non-dairy beverages. Cheeses containing greater than $320 \mathrm{mg}$ Ca per $100 \mathrm{~g}$ were classified as core dairy A (cheeses). Yoghurt and soft cheeses that contained up to $320 \mathrm{mg}$ Ca per $100 \mathrm{~g}$ were classified as core dairy B (yoghurt and soft cheeses). Cream cheese, custards, cream and dairy desserts were not classified as core dairy. If calcium data were unavailable for a given product in the database, values were estimated using the mean calcium content for products in the same food sub-category (e.g. mean of the available values of calcium content for ricotta cheeses was applied to ricotta cheeses with no calcium data). There were 682 cheese products in total and 333 (49\%) of these did not have calcium data available.

FVNL content was not available in the food database, thus estimates were made using information from ingredient lists collected online or from in-store product packaging. Food categories for which the lack or presence of FVNL data would result in a product receiving a respectively lower or higher star rating were fruits, nuts, vegetables and jams; FVNL content estimates were made for 2485 products from these food categories. Dried fruit and nut products were estimated to have $100 \%$ FVNL content. Fruit bars and jams were estimated to have $50 \%$ FVNL. Plain vegetable products were assigned $100 \%$ FVNL content (e.g. frozen vegetables). Canned or pickled vegetables were estimated to have $60 \%$ FVNL content.

Other mixed products (e.g. breakfast cereals with fruit, pizzas) that contain ingredients which contribute to FVNL points would require FVNL content of the order of $90-100 \%$ to have any impact on the rating. Using ingredient lists, it was determined that these other mixed products did not contain sufficient amounts to change the star rating, so were considered to contain $0 \%$ FVNL.

Fibre content was missing for 15339 products (76\% of the total sample). Missing fibre data was the result of it not being included on nutrition information panels and therefore not available in the database used for the present research. Many of these foods with no fibre value in the nutrition information panel are likely not a good source of fibre, such as processed meat, confectionery, oils and eggs (missing fibre data for 93\%, 94\%, 100\% and 100\% of these product categories, respectively). As fibre data were unavailable for a large proportion of the sample, products were not assigned a mean estimate value; however, this is a limitation of the study.
The HSR was calculated using the following process (also see online supplementary material, Supplemental Fig. 1 for a worked example and Supplemental Table 2 for nutrient cut-offs for the HSR):

1. FOPL Calibration Category determined.

2. Baseline points were awarded for energy, saturated fat, total sugars and sodium.

3. Modifying points were given for FVNL content, protein and fibre.

4. An overall score was calculated by subtracting modifying points from baseline points, such that a lower score would reflect a more nutritious food or drink product.

5. The HSR (from a half-star to five stars in half-star increments) was assigned based on the overall score, and FOPL Calibration and NPSC categories.

(HSR Calculator, Style Guide and Instructions available from the Health Star Rating website ${ }^{(22)}$.)

All plain waters automatically received the maximum five stars ${ }^{(22)}$.

\section{Sample}

From the original data set ( $n$ 26 509), 6284 products were excluded for any one of the following reasons: duplicated product entries arising from different package types and sizes ( $n$ 3775); missing nutrient data for all nutrients ( $n$ 2088); 'as prepared' nutrient values not available on items with 'as sold' nutrient values (primarily products such as cake mixes and powder-based meal sauces; $n$ 65); and all alcoholic beverages ( $n$ 356). Thus FOPL ratings were calculated for 20225 packaged food and drink products in the final data set.

\section{Analysis}

Analyses were conducted using the statistical software package IBM SPSS Statistics for Windows version 19·0. To address the first aim of describing how food and drink products were rated using the labelling systems, the proportion of products that received each level of star rating was reported by food category. Further analysis of the IOM three-star system was completed for dairy subcategories (cheese, cream, dairy desserts, ice cream and edible ices, milk and yoghurt) by exploring the distribution of points of low-fat compared with full-fat products. Dairy products were classified as low-fat if they contained no more than $1.5 \mathrm{~g}$ fat per $100 \mathrm{ml}$ for liquids, and $3.0 \mathrm{~g}$ fat per $100 \mathrm{~g}$ for solids ${ }^{(14)}$.

Additionally, comparisons between the two FOPL systems were drawn by converting individual product scores to a percentage of the maximum (\%Max) possible rating (e.g. $66.7 \%$ for a score of 2 out of 3 possible points in the IOM three-star system and $80 \%$ for a score of 4 out of 5 possible points in the HSR), such that the standardised scores could range from $0 \cdot 0$ to $1 \cdot 0$. Tests for normality indicated that the distributions of \%Max values at the sixteen-food-category level were non-normal, thus 
differences between the two FOPL systems with regard to the \%Max score by food category were analysed using the Wilcoxon signed-rank test for non-parametric data. The \%Max score for each food category is calculated as the median of the \%Max scores of all products in that category. These data are reported as the overall median of \%Max scores by food category.

To address the second aim of exploring FOPL system alignment with ADG, food and drink products in the database were categorised as core or discretionary using $\mathrm{ADG}^{(3)}$. Core foods were products described by the five ADG food groups: vegetables; fruit; grain/cereal foods; lean meats and substitutes; and dairy (milk, cheese, yoghurt). All other products, as well as products in these five food groups with high levels of saturated fat, added sugars or added sodium, were categorised as discretionary. Products were determined to be high in saturated fat, added sugar and/or sodium based on the list of typical foods given in the ADG as examples of discretionary choices, or similar products: ice cream, processed meats, salty crackers, sweet biscuits, cakes, jam, chocolate, cream, butter, sugarsweetened soft drinks and potato chips ${ }^{(3)}$. Additionally, the ADG recommend choosing products with sodium content less than $120 \mathrm{mg}$ per $100 \mathrm{~g}^{(3)}$. Examples of the exceptions made are sweet biscuits (grain/cereal food with high levels of saturated fat and added sugars) and frozen potato chips (vegetable food but identified in the ADG as a discretionary choice). The Mann-Whitney $U$ test was performed to explore if a difference between the median \%Max scores of core and discretionary foods and drinks existed at the broad food category level (sixteen food categories). Significance was set at $P<0 \cdot 05$.

\section{Results}

\section{Product ratings using the Institute of Medicine three-star system}

Overall, using criteria for the IOM three-star system, 11128 (55.0\%) products scored 0 points, 654 (3.2\%) scored 1 point, 3286 (16.2\%) scored 2 points and 5157 (25.5\%) scored 3 points (Fig. 3(a)). Food categories in which all products scored zero due to added sugars ineligibility were

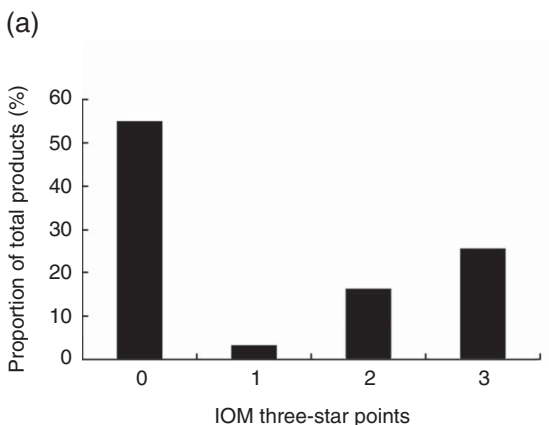

confectionery (all chocolate and lollies, jelly), dairy desserts (custard, mousse, puddings), ice cream and edible ices, jams, beverages and sugars (sugar and sweeteners, honey, dessert toppings). The majority (approximately 80\%) of packaged vegetable (e.g. frozen peas, canned legumes) and fruit (e.g. dried fruit, tinned peaches) products were granted 3 points and 2 points, respectively. Most cereal-based products also received 2 points $(24.5 \%$; e.g. fruit bread, flakebased cereal) or 3 points ( $47.3 \%$; e.g. dried plain pasta, wheat-based crispbread). More than half of milks were granted 0 or 1 point. Most cheeses and processed meats received 0 points, with 3 points being awarded to $68.1 \%$ of processed fish products and $60.7 \%$ of meat alternatives, such as tofu. Half of snack foods (e.g. crisps) received 0 points (see online supplementary material, Supplemental Table 3 for a full table of ratings by food category).

\section{Dairy}

Of the 2693 dairy products (cheeses, cream, dairy desserts, ice cream and edible ices, milk and yoghurt), 856 (31.8\%) were low-fat. The proportions of low-fat dairy products scoring 0,2 or 3 points using the IOM three-star criteria were relatively equal at $31.8 \%, 32.7 \%$ and $25.8 \%$, respectively. Fewer $(9.7 \%)$ low-fat dairy products scored 1 point under the IOM three-star system. The majority $(83.3 \%)$ of dairy products classified as full-fat scored 0 points as they exceeded the saturated fat eligbility criteria. Dairy products passing all three eligibility criteria for saturated fat, added sugars and sodium, yet not scoring the maximum 3 points did not satisfy the lower saturated fat qualifying criteria or added sugars requirements. Product ratings by dairy sub-category are shown in Supplemental Table 4 (see online supplementary material).

\section{Product ratings using the Australian Health Star Rating system}

Using the HSR algorithm, the star rating most frequently scored by products ( $n$ 3075, 15.2\%) was three-and-a-half stars from a possible five (Fig. 3(b)). Thirteen per cent of the products ( $n$ 2527) received the lowest rating of a half-star while only 833 ( $4 \cdot 1 \%$ ) scored the maximum five stars.

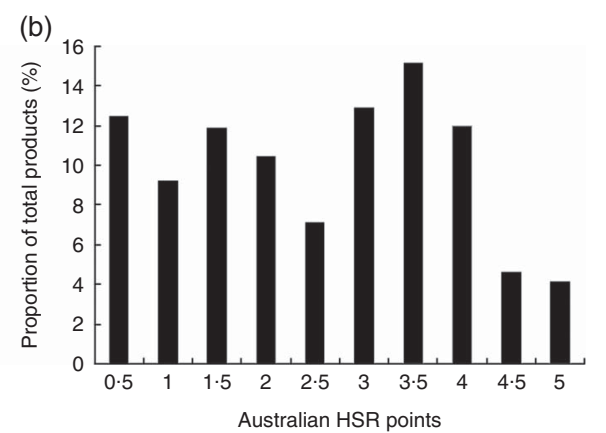

Fig. 3 Proportion (\%) of total products ( 20225 ) categorised to each scoring level using two front-of-pack labelling systems: (a) the Institute of Medicine (IOM) three-star system; (b) the Australian Health Star Rating (HSR) 
The pattern of star rating varied with food product categories. Almost all ( $95.7 \%)$ beverages scored two stars or less (see online supplementary material, Supplemental Table 5 for a full table of ratings by food category). Approximately two-thirds of vegetables received four or more stars, while three-and-a-half stars was the most common score for products in the fruit food category (38.9\% of 710 fruit products). Sixty-one per cent of milks scored four or more stars and just over half (51.8\%) of cheeses scored the minimum of a half-star. Over $80 \%$ of meat alternatives and fish products were awarded at least three-and-a-half stars $(85.5 \%$ and $83.7 \%$, respectively). Approximately half of snack foods (53.4\%) scored one-and-a-half stars or less and $51.7 \%$ of confectionery products scored the minimum rating of a half-star.

\section{Comparison of Institute of Medicine three-star and five-star Health Star Rating systems with Australian Dietary Guidelines}

Both star rating systems investigated in the present study did generally classify packaged food products in a manner that reflects ADG. For the HSR, the median \%Max score was higher for core compared with discretionary foods and drinks in all of the sixteen food categories (all $P<0.05$; Table 2). This was also true of the IOM three-star system (all $P<0.05$ ), with the exception of the fish and fish products category $(P=0 \cdot 603$; Table 3$)$.

\section{Comparison of Institute of Medicine three-star with five-star Health Star Rating system}

While both systems did reflect ADG, some differences between the average score for each food category existed when comparing the two systems (Table 4). The median \%Max score was significantly different between the FOPL systems for all except two of sixteen broad food categories - all $P$ values were $<0.05$ except for cereals and cereal products $(P=0 \cdot 347)$ and other foods $(P=0.942)$. The greatest differences in median \%Max scores between the IOM three-star and five-star HSR FOPL systems were for edible oils ( $0 \%$ and $70 \%$, respectively), convenience foods (0\% and $70 \%$ ) and dairy (0\% and $60 \%)$. Food categories with a median score of 0 using the IOM three-star criteria due to ineligibility for added sugars (beverages, confectionery, and sugars, honey and related products) also scored relatively poorly using the HSR criteria. The lowest of these median \%Max scores using the HSR criteria was $30 \%$ for beverages, $10 \%$ for confectionery and $20 \%$ for sugars.

\section{Discussion}

FOPL should provide a summary of the nutritional quality of a food that is easily interpreted at the point of purchase and is consistent with dietary guidelines. The results of the present study show that the IOM three-star and Australian HSR systems do rate foods and drinks in such a way as to be consistent with the messages of the $\mathrm{ADG}^{(3)}$. These results also show that, at a broad food category level, there is a good agreement in how the healthiness of foods is rated by both systems, although some differences in ratings were seen among dairy products and convenience foods. Largely, differences between the two systems are a result of strict eligibility criteria using the three-star system,

Table 2 Comparison of \%Max ratings by core and discretionary foods, Australian Health Star Rating†

\begin{tabular}{|c|c|c|c|c|c|c|}
\hline \multirow[b]{3}{*}{ Food category } & & & \multicolumn{4}{|c|}{$\%$ Max score } \\
\hline & \multicolumn{2}{|c|}{ Number } & \multicolumn{2}{|c|}{ Core } & \multicolumn{2}{|c|}{ Discretionary } \\
\hline & Core & Discretionary & Median & IQR & Median & IQR \\
\hline Bread and bakery products & 761 & 1668 & $70^{*}$ & $10-100$ & $30^{*}$ & $10-100$ \\
\hline Cereal and cereal products & 1244 & 699 & $80^{*}$ & $10-100$ & $60^{*}$ & $10-100$ \\
\hline Confectionery & 0 & 1434 & \multicolumn{2}{|c|}{-} & 10 & $10-100$ \\
\hline Convenience foods & 88 & 986 & $60 \ddagger$ & $40-100$ & $70 \ddagger$ & $10-100$ \\
\hline Dairy & 1716 & 977 & $65^{\star}$ & $0-100$ & $50^{*}$ & $0-100$ \\
\hline Edible oils and emulsions & 0 & 482 & & & 70 & $10-100$ \\
\hline Eggs & 96 & 0 & 80 & $80-80$ & \multicolumn{2}{|c|}{-} \\
\hline Fish and fish products & 554 & 139 & $80^{*}$ & $10-90$ & $70^{*}$ & $30-90$ \\
\hline Fruit and vegetables & 2055 & 621 & $80^{*}$ & $10-100$ & $40^{*}$ & $10-100$ \\
\hline Meat and meat products & 162 & 770 & $80^{*}$ & $10-100$ & $40^{*}$ & $10-100$ \\
\hline Non-alcoholic beverages & 0 & 1847 & \multicolumn{2}{|c|}{-} & 30 & $10-100$ \\
\hline Sauces and spreads & 0 & 2340 & \multicolumn{2}{|c|}{-} & 40 & $10-100$ \\
\hline Snack foods & 0 & 639 & \multicolumn{2}{|c|}{-} & 30 & $10-100$ \\
\hline Sugars, honey and related products & 0 & 334 & \multicolumn{2}{|c|}{-} & 20 & $10-100$ \\
\hline Special foods & 236 & 101 & $70^{*}$ & 10-90 & $20^{*}$ & $10-100$ \\
\hline Other foods & 0 & 276 & \multicolumn{2}{|c|}{-} & 70 & $10-100$ \\
\hline Total sample & 6912 & 13313 & $80^{*}$ & $10-100$ & $40^{*}$ & $10-100$ \\
\hline
\end{tabular}

$\mathrm{IQR}$, interquartile range.

${ }^{*} P<0.001$; Mann-Whitney $U$ test.

tIndividual product scores were converted to a percentage of the maximum possible rating (e.g. $66.7 \%$ for a rating of 2 out of 3 possible points in the Institute of Medicine three-star system and $80 \%$ for a rating of 4 out of 5 possible points in the Australian Health Star Rating).

$\ddagger P=0.097$. 
Table 3 Comparison of \%Max ratings by core and discretionary foods, Institute of Medicine three-star system†

\begin{tabular}{|c|c|c|c|c|c|c|}
\hline \multirow[b]{3}{*}{ Food category } & & & \multicolumn{4}{|c|}{$\%$ Max score } \\
\hline & \multicolumn{2}{|c|}{ Number } & \multicolumn{2}{|c|}{ Core } & \multicolumn{2}{|c|}{ Discretionary } \\
\hline & Core & Discretionary & Median & IQR & Median & IQR \\
\hline Bread and bakery products & 761 & 1668 & $100^{*}$ & $0-100$ & $33 \cdot 3^{*}$ & $0-100$ \\
\hline Cereal and cereal products & 1244 & 699 & $100^{*}$ & $0-100$ & $0^{*}$ & $0-100$ \\
\hline Confectionery & 0 & 1434 & & 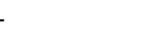 & 0 & $0-0$ \\
\hline Convenience foods & 88 & 986 & $66 \cdot 7^{\star}$ & $00-100$ & $0^{*}$ & $0-100$ \\
\hline Dairy & 1716 & 977 & $0^{*}$ & $0-100$ & $0^{*}$ & $0-100$ \\
\hline Edible oils and emulsions & 0 & 482 & & ( & 0 & $0-100$ \\
\hline Eggs & 96 & 0 & $66 \cdot 7$ & $66 \cdot 7-66 \cdot 7$ & & \\
\hline Fish and fish products & 554 & 139 & $100 \ddagger$ & $0-100$ & $100 \ddagger$ & $0-100$ \\
\hline Fruit and vegetables & 2055 & 621 & $66 \cdot 7^{\star}$ & $0-100$ & $0^{*}$ & $0-100$ \\
\hline Meat and meat products & 162 & 770 & $0^{*}$ & $0-100$ & $100^{*}$ & $0-100$ \\
\hline Non-alcoholic beverages & 0 & 1847 & & - & 0 & $0-0$ \\
\hline Sauces and spreads & 0 & 2340 & & - & $66 \cdot 7$ & $0-100$ \\
\hline Snack foods & 0 & 639 & & - & 0 & $0-100$ \\
\hline Sugars, honey and related products & 0 & 334 & & - & 0 & $0-0$ \\
\hline Special foods & 236 & 101 & $66 \cdot 7^{\star}$ & $33 \cdot 3-100$ & $66 \cdot 7^{*}$ & $0-100$ \\
\hline Other foods & 0 & 276 & & 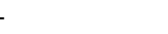 & $66 \cdot 7$ & $0-100$ \\
\hline Total sample & 6912 & 13313 & $66 \cdot 7^{\star}$ & $0-100$ & $0^{*}$ & $0-100$ \\
\hline
\end{tabular}

$\mathrm{IQR}$, interquartile range.

${ }^{*} P<0.001$; Mann-Whitney $U$ test.

†Individual product scores were converted to a percentage of the maximum possible rating (e.g. $66.7 \%$ for a rating of 2 out of 3 possible points in the Institute of Medicine three-star system and $80 \%$ for a rating of 4 out of 5 possible points in the Australian Health Star Rating).

$\ddagger P=0.860$.

Table 4 Comparison of two nutrient assessment systems by median \%Max food category score†

\begin{tabular}{|c|c|c|c|c|c|}
\hline \multirow[b]{2}{*}{ Food category } & \multicolumn{2}{|c|}{$\%$ Max score for IOM three-star system $\ddagger$} & \multicolumn{2}{|c|}{ \%Max score for Australian HSR§ } & \multirow[b]{2}{*}{$P \|$} \\
\hline & Median & IQR & Median & IQR & \\
\hline Breads and bakery products ( $n$ 2249) & $66 \cdot 7$ & $0-100$ & 40 & $20-70$ & $<0.001$ \\
\hline Cereals and cereal products ( $n$ 1943) & $66 \cdot 7$ & $33 \cdot 3-100$ & 70 & $60-80$ & 0.347 \\
\hline Confectionery ( $n$ 1434) & 0 & $0-0$ & 10 & $10-30$ & $<0.001$ \\
\hline Convenience foods $\Phi(n$ 1074) & 0 & $0-0$ & 70 & $60-70$ & $<0.001$ \\
\hline Dairy ( $n$ 2693) & 0 & $0-66.7$ & 60 & $30-70$ & $<0.001$ \\
\hline Edible oils and oil emulsions ( $n$ 482) & 0 & $0-0$ & 70 & $57 \cdot 5-70$ & $<0.001$ \\
\hline Eggs $(n 96)$ & $66 \cdot 7$ & $66 \cdot 7-66 \cdot 7$ & 80 & $80-80$ & $<0.001$ \\
\hline Fish and fish products ( $n 693$ ) & 100 & $66 \cdot 7-100$ & 80 & $70-80$ & $<0.001$ \\
\hline Fruit and vegetables ( $n 2676)$ & 66.7 & $0-100$ & 70 & $50-80$ & $<0.001$ \\
\hline Meat and meat products ( $n$ 932) & 0 & $0-66.7$ & 40 & $30-70$ & $<0.001$ \\
\hline Non-alcoholic beverages ( $n$ 1847) & 0 & $0-0$ & 30 & $20-30$ & $<0.001$ \\
\hline Sauces and spreadstt (n 2340) & 66.7 & $0-100$ & 40 & $30-60$ & 0.046 \\
\hline 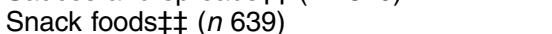 & 0 & $0-100$ & 30 & $20-50$ & $<0.001$ \\
\hline Sugars, honey and related products ( $n 334$ ) & 0 & $0-0$ & 20 & $20-30$ & $<0.001$ \\
\hline Special foods $\S \S(n$ 337) & $66 \cdot 7$ & $66 \cdot 7-100$ & 70 & $50-70$ & $<0.001$ \\
\hline Other foodsIIII ( $n$ 276) & $66 \cdot 7$ & $33 \cdot 3-100$ & 70 & $40-90$ & 0.942 \\
\hline
\end{tabular}

IOM, Institute of Medicine; HSR, Health Star Rating.

tIndividual product scores were converted to a percentage of the maximum possible rating (e.g. $66.7 \%$ for a rating of 2 out of 3 possible points in the IOM three-star system and $80 \%$ for a rating of 4 out of 5 possible points in the Australian HSR).

$\ddagger$ Median percentage of maximum score of the total sample for the IOM three-star system was $0 \%$ (zero stars)

§Median percentage of maximum score of the total sample for the Australian HSR system was $50 \%$ (approximately two-and-a-half stars).

IIWilcoxon signed-rank test.

ПConvenience foods: pizza, sandwiches, salads, ready meals, meal kits.

††Sauces and spreads: salad dressings, cooking sauces, savoury and sweet spreads.

抽ack foods: potato crisps, corn chips, extruded snacks.

$\S \S$ Special foods: diet meal replacements, breakfast beverages, baby foods.

IIIIOther foods: unable to be categorised into any other food category.

meaning that many products scored zero stars, and the different range of nutrients assessed by each model.

Of interest is that when categorised as a core or discretionary food or drink using $\mathrm{ADG}^{(3)}, 65 \cdot 8 \%$ of products were considered to be discretionary. This is an important public health message, as the majority of all products available in supermarkets do not support a healthy, well-balanced diet. Also noteworthy is that one-fifth of the sample was ineligible to receive points using the IOM three-star system due to the added sugars criteria which are applied to whole food categories rather than individual products, reflecting that $20 \%$ of the Australian food supply 
is high in sugars. This supports the ADG which advocate for fresh, unpackaged foods to form the foundation of a nutritious diet.

Despite dietary guidelines promoting the consumption of whole, minimally processed foods, consumers are increasingly presented with an overabundance of packaged food products on supermarket shelves, as is evidenced by the tens of thousands of products included in the database used for the current study. Australian legislation requires that these products display nutrition information in the form of a nutrition information panel; however, these panels are limited when consumer understanding is considered $^{(5)}$. Similarly, the Daily Intake Guide currently displayed on many Australian products has been shown to be less effective at enabling consumers to identify healthier products when compared with labelling formats containing an interpretive element such as colour-coded traffic lights ${ }^{(8,12)}$. Following a review process, the HSR was announced to be implemented in Australia as the preferred FOPL format ${ }^{(13)}$.

No other studies have compared the validity of the Australian HSR with any other FOPL or nutrient profile model. There is, however, evidence supporting the use of interpretive FOPL systems such as star ratings or Traffic Light labelling in preference to the Daily Intake Guide and nutrition information panels for enabling consumers to determine healthier products ${ }^{(8,12,23,24)}$. More extensive validity testing has been undertaken on the WXYfm model and $\mathrm{NuVal}^{(25-27)}$.

The two star-based FOPL systems in the present study both utilise across-the-board ratings, meaning that all foods in the database receive a rating that is comparable to all other products, rather than within-category scores ${ }^{(28)}$. Across the total sample of products, the rating for all core foods using both FOPL systems was significantly higher than for discretionary foods. This was also true for each of the five core food groups of the ADG. For example, the vegetables category of the database includes plain frozen vegetables (core) and frozen potato chips (discretionary). Consistent with ADG, core vegetables were awarded higher ratings than discretionary frozen vegetables. This suggests that both systems will allow consumers to identify healthier food choices within product categories as well as across the board as the systems are designed. However, the spread of possible scores using the HSR is greater than that of the IOM three-star system as products can receive from a half-star up to five stars in half-star increments, in comparison to zero to three stars in whole-star increments. This greater range may be more useful for consumers as it enables product diversity to be better shown.

At the individual product level however, there appeared to be some anomalies. Ninety (0.7\%) of 13313 discretionary foods and drinks scored the maximum of five stars using HSR criteria. Most of these were diet-related products such as protein bars and drinks, as well as sugar-free confectionery and some cooking oils. Using the IOM three-star criteria, 1927 (14.5\%) discretionary foods and drinks scored the maximum of 3 points. These items included diet-related products, snack foods (crisps, popcorn), ready meals/convenience foods, and savoury sauces and spreads. One potential reason for this is that both rating systems are based on a limited range of nutrients. For example, using the HSR system eight confectionery items (sugar-free pastilles) scored five stars and only thirteen fruit products (e.g. dried fruit and nuts) also scored five stars. These confectionery items have low energy, saturated fat, total sugars and sodium, and approximately double the amount of fibre per $100 \mathrm{~g}$ as the fruit products that scored five stars.

This unintended classification of some products may challenge the construct validity in nutrient profiling ${ }^{(29)}$. While the sugar-free pastilles were portrayed as healthy products by the HSR, they would be unable to realistically constitute a healthy diet as they lack many of the nutrients vital to human health, thus resulting in confusing public health nutrition messages. Rayner et al. identify the various methods for validating nutrient profile models and that the method used in the present paper, whereby product ratings were compared with food-based dietary guidelines, is most useful in the early stages of model development ${ }^{(30)}$. The current study was conducted at a time when the HSR calculator was in draft form and as such the identification of any outstanding anomalies may have been useful for improving the accuracy of the model. Further validation of the IOM three-star and final HSR models could be strengthened by undertaking another of the validation methods such as comparing food ratings with nutrition experts' views, or with ideal 'healthy' diets ${ }^{(30)}$.

A strength of the HSR is its underlying use of the NPSC, which is used to regulate food claims in Australia. The NPSC model is a modified version of the WXYfm or Ofcom model for regulating food advertisements in the UK on which more extensive validation work has been conducted ${ }^{(25,27,28)}$. Another study using the validation method of comparing a nutrient profiling model with dietary guidelines has been conducted on the WXYfm model ${ }^{(25)}$. As with the present study, the WXYfm model was shown to categorise foods in line with the UK's national dietary recommendations ${ }^{(25)}$.

One strength of the HSR over the IOM three-star system is the assessment of plain water. For the IOM three-star system, the classification of water as a beverage resulted in it being ineligible for points as per the added sugars criteria, despite it containing no added sugars. Thus it would carry the same FOPL rating as sugar-sweetened beverages, contradicting the message of the ADG to consume water in preference to sweetened beverages ${ }^{(3)}$. Within the HSR, the clause that all plain waters receive the maximum rating of five stars is an important one that creates consistency between FOPL and dietary guidelines. A potential solution to this issue could be for the IOM three-star system to also include a special condition for water to receive maximum points. Another point of distinction between the two systems is the relative ratings that are given to dairy products. In each of the dairy 
sub-categories (milk, cheese and other dairy), the median \%Max score for the HSR was at least double that of the IOM three-star system. For example, some reduced-fat milks received \%Max scores of $0 \%$ or $33 \%$ using three-star criteria (equivalent to zero and one star) but as high as 70-100 \% using the HSR (equivalent to three-and-a-half to five stars). Such a difference was expected as the HSR incorporates special consideration to core dairy foods while the IOM three-star system treats dairy products the same as all other foods.

There were two food categories for which the differing use of per serving and per $100 \mathrm{~g}$ by the two FOPL systems may have influenced the resulting product rating: convenience foods and snack foods. For convenience foods (e.g. pizza, pre-packaged sandwiches, soups, microwaveable ready meals), the low median \%Max score using IOM three-star criteria was a result of $82 \%$ of products not meeting the eligibility criteria for sodium and thus scoring zero stars. Almost $90 \%$ of convenience foods had a labelled serving size of $100 \mathrm{~g}$ or greater and so the sodium content per serving far exceeded the eligibility criteria. For snack foods, the amounts of saturated fat and sodium with a relatively small serving size ( $93 \%$ of snack foods had a labelled serving size $\leq 50 \mathrm{~g}$ ) were lower than the eligibility cut-offs of the IOM three-star criteria. This issue of assessment per $100 \mathrm{~g}$ or per serving highlights that without an agreed standard serving size for foods and drinks, there is potential to achieve a higher IOM three-star rating by manipulating the serving size listed on a product.

Issues such as this highlight the importance of a comprehensive approach to introducing FOPL, including public education campaigns to ensure consumers can correctly interpret the information, regardless of the format used. Furthermore, the impact that voluntary or mandatory implementation of the FOPL will have on uptake by industry should be considered. A voluntary FOPL system using the IOM three-star criteria could be confusing to consumers as products that do not display any star could either be ineligible because of high saturated fat, sugar and/or sodium or due to its manufacturer deciding not to participate in the FOPL system. In contrast, foods using the HSR system would carry at least a half-star and thus a product with no stars clearly indicates its manufacturer's non-participation. Previous Australian and international research has shown that a voluntary approach to FOPL resulted in inconsistent implementation ${ }^{(31,32)}$. For this reason, a mandatory standard FOPL would almost certainly better enable consumers to evaluate the nutritional quality of foods.

The results of the present research should be interpreted with caution due to several limitations. First, the food database information was recorded from and depends on the accuracy of the nutrition information panel on the food label. However, any FOPL system is likely to be based on values from the nutrition panel. The cause of any discrepancies between the FOPL information and actual nutrient content is likely due to inaccurate reporting by manufacturers and not arising from the database itself. Second, the use of blanket estimates by food category for added sugars was required as these data are not routinely available in nutrient databases or presented on food labels in Australia. Similarly, data were not available for FVNL content and thus estimates were made for the fruit and vegetable food categories but not for mixed dishes containing fruit, vegetables, nuts or legumes (e.g. convenience foods, breakfast cereals). Trials with a selection of mixed dishes indicated that the ratings for these products did not change when FNVL was or was not included as they did not contain a sufficient proportion of FVNL to have any effect.

Despite these limitations, the overall results of the study do indicate that these two FOPL systems would provide consumers with summary nutritional information that is consistent with national dietary guidelines. This is true of the Australian setting; however, further research would be required to examine if this is also the case for food supplies and dietary guidelines in other countries.

\section{Conclusion}

Front-of-pack nutrition labelling should enable consumers to identify foods that contribute to healthier diets. As assessed in the present study, both the IOM three-star and HSR FOPL systems categorised packaged foods and drinks broadly in line with ADG to consume a variety of vegetables, fruit, cereal products, lean protein foods and dairy. Differences in food category ratings between the two systems arise from the strict eligibility criteria of the threestar system, which automatically excludes some foods, and the different nutrients assessed by each model. The greater spread in the range of scores using the HSR system may allow consumers to better determine the relative health value of packaged food and drink products in comparison to the IOM three-star system.

\section{Acknowledgements}

Financial support: This research received no specific grant from any funding agency in the public, commercial or not-for-profit sectors. Conflict of interest: None. Authorship: A.M.C. and J.C.Y.L. designed the research; A.M.C. and J.C.Y.L. conducted the research; H.R.Y., E.K.D. and B.C.N. provided essential materials; A.M.C. analysed the data; A.M.C., J.C.Y.L., H.R.Y., E.K.D., B.C.N. and V.M.F. wrote the paper; A.M.C. had primary responsibility for final content. All authors read and approved the final manuscript. Ethics of human subject participation: Not applicable.

\section{Supplementary material}

To view supplementary material for this article, please visit http://dx.doi.org/10.1017/S1368980015002748 


\section{References}

1. Ng M, Fleming T, Robinson M et al. (2014) Global, regional, and national prevalence of overweight and obesity in children and adults during 1980-2013: a systematic analysis for the Global Burden of Disease Study 2013. Lancet 384, 766-781.

2. World Health Organization (2003) Diet, Nutrition and the Prevention of Chronic Diseases. Joint WHO/FAO Expert Consultation. WHO Technical Report Series no. 916. Geneva: WHO.

3. National Health and Medical Research Council (2013) Australian Dietary Guidelines. Canberra: NHMRC.

4. Food Standards Australia New Zealand (2011) Overview and Application of Food Labelling and Information Requirements: User Guide to Standard 1.2.1 - Labelling and Other Information Requirements. Canberra: FSANZ.

5. Cowburn G \& Stockley L (2005) Consumer understanding and use of nutrition labelling: a systematic review. Public Health Nutr 8, 21-28.

6. Lobstein T \& Davies S (2009) Defining and labelling 'healthy' and 'unhealthy' food. Public Health Nutr 12, 331-340.

7. Hawley K, Roberto C, Bragg M et al. (2013) The science of front-of-package food labels. Public Health Nutr 16, 430-439.

8. Kelly B, Hughes C, Chapman K et al. (2009) Consumer testing of the acceptability and effectiveness of front-of-pack food labelling systems for the Australian grocery market. Health Promot Int 24, 120-129.

9. Australian Food and Grocery Council (2011) Daily Intake Guide. http://www.mydailyintake.net (accessed March 2013).

10. National Heart Foundation of Australia (2013) Heart Foundation Tick. http://www.heartfoundation.org.au/healthyeating/heart-foundation-tick/Pages/default.aspx (accessed March 2013).

11. Blewett N, Goddard N, Pettigrew S et al. (2011) Labelling Logic: Review of Food Labelling Law and Policy 2011. Canberra: Commonwealth of Australia.

12. Borgmeier I \& Westenhoefer J (2009) Impact of different food label formats on healthiness evaluation and food choice of consumers: a randomized-controlled study. $B M C$ Public Health 9, 184.

13. Australian Government Department of Health (2013) Frontof-pack labelling updates. https://www.health.gov.au/ internet/main/publishing.nsf/Content/foodsecretariat-frontof-pack-labelling-1 (accessed June 2013).

14. Food Standards Australia New Zealand (2013) Standard 1.2.7 - Nutrition, Health and Related Claims. Canberra: FSANZ.

15. Institute of Medicine (2012) Front-of-Package Nutrition Rating Systems and Symbols: Promoting Healthier Choices. Washington, DC: The National Academies Press.

16. World Health Organization (2010) Nutrient Profiling: Report of a WHO/IASO Technical Meeting. Geneva: WHO.
17. The George Institute for Global Health (2013) FoodSwitch. http://www.georgeinstitute.org.au/foodswitch (accessed March 2013).

18. Julia C, Kesse-Guyot E, Touvier M et al. (2014) Application of the British Food Standards Agency nutrient profiling system in a French food composition database. Br J Nutr 112, 1699-1705.

19. NSW Food Authority (2009) Report on 2009 Trans Fatty Acid Survey - Analytical Results. Silverwater: NSW Food Authority.

20. Food Standards Australia New Zealand (2009) Review Report: Trans Fatty Acid in the New Zealand and Australian Food Supply. Canberra: FSANZ.

21. Louie JCY, Moshtaghian H, Boylan S et al. (2015) A systematic methodology to estimate added sugar content of foods. Eur J Clin Nutr 69, 154-161.

22. Commonwealth of Australia (2014) Health Star Rating System Calculator and Artwork. http://healthstarrating.gov. $\mathrm{au} /$ internet/healthstarrating/publishing.nsf/Content/calculator (accessed September 2015).

23. Maubach N, Hoek J \& Mather D (2014) Interpretive front-ofpack nutrition labels. Comparing competing recommendations. Appetite 82, 67-77.

24. Watson W, Kelly B, Hector D et al. (2014) Can front-of-pack labelling schemes guide healthier food choices? Australian shoppers' responses to seven labelling formats. Appetite $\mathbf{7 2}$, 90-97.

25. Arambepola C, Scarborough P \& Rayner M (2008) Validating a nutrient profile model. Public Health Nutr 11, 371-378.

26. Katz D, Njike V, Rhee L et al. (2010) Performance characteristics of NuVal and the Overall Nutritional Quality Index (ONQI). Am J Clin Nutr 91, issue 4, 1102S-1108S.

27. Scarborough P, Boxer A, Rayner M et al. (2007) Testing nutrient profile models using data from a survey of nutrition professionals. Public Health Nutr 10, 337-345.

28. Scarborough P, Arambepola C, Kaur A et al. (2010) Should nutrient profile models be 'category specific' or 'across the board'? A comparison of the two systems using diets of British adults. Eur J Clin Nutr 64, 553-560.

29. Clerfeuille E, Vieux F, Lluch A et al. (2013) Assessing the construct validity of five nutrient profiling systems using diet modeling with linear programming. Eur J Clin Nutr 67, 1003-1005.

30. Rayner M, Scarborough P \& Kaur A (2013) Nutrient profiling and the regulation of marketing to children. Possibilities and pitfalls. Appetite 62, 232-235.

31. Carter OBJ, Mills BW, Lloyd E et al. (2013) An independent audit of the Australian food industry's voluntary front-ofpack nutrition labelling scheme for energy-dense nutritionpoor foods. Eur J Clin Nutr 67, 31-35.

32. Van Camp D, de Souza Monteiro DM \& Hooker NH (2012) Stop or go? How is the UK food industry responding to front-of-pack nutrition labels? Eur Rev Agric Econ 39, 821-842. 